\title{
Postoperative Biliary Peritonitis
}

\author{
Shaposhnikov Veniamin Ivanovich*
}

*Department of Surgical diseases, Russia

*Corresponding author: Shaposhnikov Veniamin Ivanovich, Department of Surgical diseases, Russia.

\begin{abstract}
Annotation
The author watched 83 patients with postoperative peritonitis bilious, of whom $27(32.5 \%)$ the reason for its development was inspired by the ongoing peritonitis, $38(45.9 \%)$ is non-suite Lodge of the gall bladder, $3(3.6 \%)$-damage to abnormally developed bile ducts, 8 (9.6\%)-migration tubes exegesis, 7 (8.4\%)-wound exegesis. Died 8 (9.6\%) patients. The author notes the difficulty of diagnosis of this complication due to the paucity of symptoms.
\end{abstract}

Keywords: Operation; complication; bile peritonitis; diagnosis; treatment

\section{Introduction}

Lately there has been a significant increase in patients with acute cholecystitis, if this fatality rate when it is 3-7\% [1]. Analysis of literature data suggests that even now, despite great technical capabilities of modern surgery, surgeries for cholecystitis are accompanied by various tactical and technical medical errors. So, if damage reaches exegesis cholecystectomy $5.6 \%$ and zhelcheistechenie from gallbladder bed is $8.3 \%$ [2]. In 68\%$81.1 \%$ of cases the reason for the development of postoperative biliary peritonitis are tactical and technical medical errors $[3,4,5]$. Prominent negative role for prediction of treatment provides and the presence of patients expressed disorders of immunity and slowing the regeneration process [5]. The most frequent complications arise during surgery in patients with destructive form of this disease, the syndrome of Mirizzi, sclerosis of the gallbladder and biliary tract abnormalities. Postoperative mortality in persons aged patients reaches $80 \%$ [2.5]. Prognosis worsens or if there already or developing in the patient of acute pancreatitis. Such observations lethality reaches $30-35 \%$ [1]. These data demonstrate the feasibility of evaluating the treatment of patients with acute cholecystitis in the various hospitals to develop a diagnostic and treatment algorithm specified complications.

\section{Material and Methods}

The OCG in surgical units were MUNICIPAL treatment 5412 patients with acute cholecystitis. Men (33.7\%), 1828 and women-3584 (66.3\%). the age of the patients was from 21 until
95 years. Operations are performed at 4106 (75.8\%) patients, among them a laparoscopic way-from 1728 (42.1\%), conversion option-250 (6.1\%) Postoperative lethality was $2.18 \%$. All patients, including those under the age of 50 years, revealed on 2 or more For Co morbidities. Emergency operations were performed at 2464 (60\%) patients. Destructive form of inflammation identified at $3116(75.9 \%)$ patients and it has always been associated with the more or less pronounced inflammatory infiltrate around the gallbladder with involvement in the pathological process of adjacent liver tissue and liver the duodenum bundles, with 507 (14.8\%) He was a development density. Out of the total number of cholecystectomy (4106) postoperative biliary peritonitis, varying degrees of prevalence was observed in $83(2.02 \%)$ patients. At 8 $(0.19 \%)$ of them he was the cause of death.

A complication they had revealed before relaparotomy and confirmed during the repeated surgical intervention. For the diagnosis of this complication conducted clinical and laboratory, biochemical and instrumental examinations, including ultrasound, CT scan, x-ray and laparoscopy, abdominal and thoracic cavities. From 83 patients were carried out at relaparotomy 22 (26.5\%) of them have 18 with symptoms of widespread peritonitis and 4 after random enforced pulling the drain tube from the patient when turning-exegesis nabob (among these patients during relaparotomy discovered a clump of bile under the liver). At 41 (49.4\%) same patient relaparotomy was replaced by venting bile accumulation zones (in the right podreberie, right side canal and pelvic cavity) 
under ultrasound control. Reasons for the development of postoperative bile peritonitis were: continuing peritonitis-27 $(32.5 \%)$, non-suite lodge gallbladder-38 (45.9\%), corruption of abnormally developed bile ducts-3 (3.6\%), the migration of the drainage tube from exeresis-8 (9.6\%), wound choledochitis-7 (8.4\%) On clinical flow disease from 83 patients, only 12 (14.4\%) There were symptoms of peritonitis, and 71 (85.6\%)-they were erased.

On the prevalence of abdominal lesion bed (limited and unlimited) peritonitis diagnosed at 65 (78.3\%), and common-u 18 (21.7\%) should indicate if local peritonitis symptoms of peritoneal sepsis was observed then when they were distributed in all patients, and $5(6 \%)$ He even accompanied the infectious-toxic shock (these patients died). Marked by a certain relationship between severity of endogenous intoxication and volume zhelcheistechenija. So, if you lose your 300-500 ml of bile develops local peritonitis with mild degree of intoxication, 500-1000 ml-diffusive-spilled with average and more than $1000 \mathrm{ml}-\mathrm{General}$ severe peritonitis. From 83 patients with Leukocytosis, with a shift of Leukocyte formula left, there were only 31 (37.3\%). Thus, in the majority of patients leading diagnostic test was abundant expiration of bile from the abdominal cavity, or drainage, or past them.

\section{Results}

5412 of patients with acute cholecystitis (1.66\%) and 90 died people from postoperative bile peritonitis $8(0.16 \%)$. The remaining 82 patients died from other causes. From 83 patients with postoperative peritonitis bilious died 8 (9.6\%) of the 27 patients with peritonitis was before surgery and he continued after she died 4 (15\%), conditionally from 38 patients with nonushitym gallbladder bed-1 (2.6\%) From 3 patients where the damage occurred an abnormally developed bile ducts, there have been no deaths. Of the 8 patients who died of the drainage tube migration, died 1 (12.5\%), conditionally of 7 patients with wound choledochitis died 2 (28.5\%) of probation. We believe it necessary to point out that damage to abnormally developed bile ducts and was immediately identified exeresis wound during the execution of the primary operation and steps were taken to restore the tightness of biliary tract, bile peritonitis but evolved, Despite the timely diagnosis of this complication. All 10 patients were performed relaparotomy during which only 8 have external drainage of biliary tract, and the 2-x stitches on holedoh, but after 1 day they prorezalis, accompanied by a progression of his fatal peritonitis exodus.

\section{Discussion}

Thus, the development of postoperative bile peritonitis was due to tactical and technical medical mistakes made as when choosing how to gall bladder removal and manipulation on this body and its surrounding tissues, and also when draining the abdominal cavity. A negative role played and the older patient's violations of immunity. If you get the drain tube from the exegesis, diagnose the development of this complication did not pose any difficulty, when his other reasons it was difficult-because of wear of clinical symptoms. This can be attributed to the conduct of postoperative corrective medical therapy, use of antibiotics and pain medication. However, the patients symptoms of intoxication (tachycardia and dehydration) and euphoria, but the main thing-the expiration of bile by drainage tubes, allowed or suspect the complications, or conclude the progression of peritonitis if he had before the surgery. Severity of the pathological process is largely dependent on zhelcheistechenija. So the lowest mortality was observed in patients with defects in the closing of the lodge of the gall bladder.

It is possible to explain the cover bed great seal and other soft tissues, limiting the speed of zhelcheistechenija. Rate and amount of bile in the free abdomen mostly depend on the diameter and the nature of the damage to the bile duct, as well as on the availability of occlusion is below the level of his trauma. The greater was hypertension, the faster evolved intoxication, with 8 (9.6\%) patient's zhelcheistechenie to drain the tubes should not exceed $50 \mathrm{ml}$, and took place by them. Effaced symptomatology was the main reason the diagnosis on complications lag 1-2 days. With the increase in the number of zhelcheistechenija is progressing not only peritonitis, but endogenous toxemia. In such cases, the expectant surgical treatment becomes dangerous to the life of the patient. However, the mere relaparatomy or drainage of abdominal cavity under ultrasound control does not ensure success in the treatment of this complication. To do this, you must use complex medication therapy, which helps secure the correction vodno-elektrolitnogo balance, renal and hepatic failure to conduct the fight against toxemias and microbial aggression.

\section{Conclusion}

Postoperative biliary peritonitis usually driven by technical and tactical mistakes when performing cholecystectomy. Most frequently surgeons admit negligence when closing a lodge of the gall bladder. Rough pricking the surrounding liver tissue accompanied by damage to needle close located vnutripechenerngo bile duct that is accompanied by the expiration of bile into the abdominal cavity-free. In such cases rarely imposed the seams do not provide impermeability Lodge. Risk of bile peritonitis increases dramatically when injury anomalous biliar no traumatic ducts. For this reason, when determining the patient's development anomalies Brigade must immediately be turned surgeon, has extensive experience in biliary tract surgery. Particular nuisance calls cause gall development of peritonitis such as migration of the tube, which the surgeon introduced clearance exegeses. Most often this occurs when the patient is rotated on its side. However, it tube prolapsed may occur and when the surgeon does not take into account its length from exegeses and up to the abdominal wall.

If it is short, when awakening the patient strains, inflates, twitches, and as a result dramatically increases the distance from exegesis and to the anterior abdominal wall and tube vydjorgivaetsja 
of duct, that is to create a vnutribrjushinnyj supply of its length. Wound choledochitis usually occurs when a patient has a dense inflammatory infiltrate. Of particular concern is the incidence of severe biliary peritonitis while destructive-nekroticheskom lesions of gallbladder and pancreas. These patients require not only adequate drainage of the abdominal cavity, but also sound therapy and antifermentnuju is pathogenetically local hypothermia pancreas.

\section{References}

1. Krasilnikov DM , Farrahov AZ , Khairullin II , Mavrin MI (2008) Early postoperative complications in patients and calculous cholecystitis holedoholitiazom. Kazan M, pp. 154.

(c) ()

This work is licensed under Creative Commons Attribution 4.0 License

To Submit Your Article Click Here: Submit Article

DOI: $10.32474 /$ SCSOAJ.2019.02.000141
2. Charyshkin Al, Midlenko OV (2009) Way cholecystectomy in complicated forms of acute cholecystitis. Herald of surgical Gastroenterology 2: 5659.

3. Efimenko (1994) N.I. Postoperative peritonitis: atoref. Dees. Dr. med. Sciences, p. 24.

4. Nichitajlo ME, Skums AV (2006) Bile duct Injury during cholecystectomy and its consequences. Kiev Poppy seeds, pp. 343.

5. Daminova NM, Kurbonov KM, Sharipov H Ju, Sadullov DN (2008) Reasons for and methods of integrated treatment of postoperative biliary peritonitis. Harkivska hirurgichna school 2: 158-163.

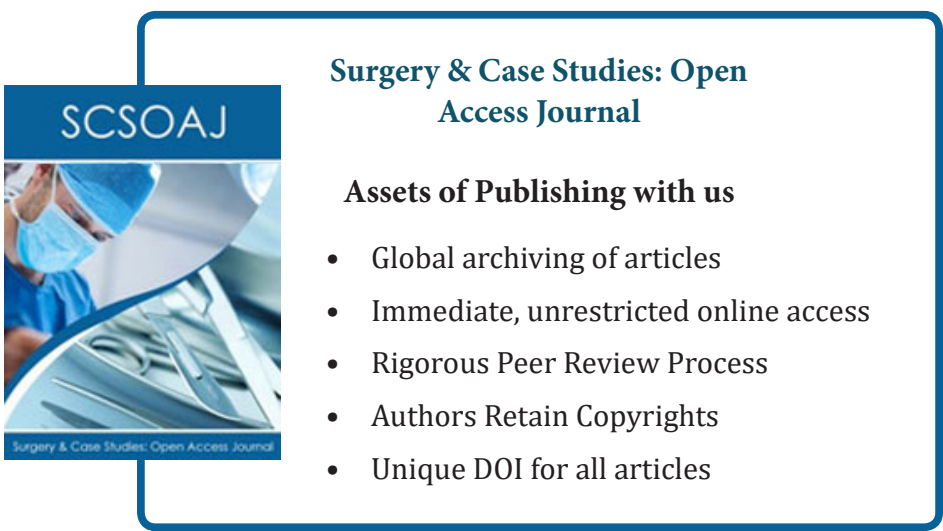

